\title{
Accurate Results for Free Vibration of Doubly Curved Shallow Shells of Rectangular Planform (Part 1)
}

\author{
Daisuke Narita ${ }^{\mathrm{a}, *}$, Yoshihiro Narita ${ }^{\mathrm{b}}$ \\ ${ }^{a}$ Hokkaido University of Science Junior College, Teineku, Sapporo, Japan. Email: narita@ hus.ac.jp \\ bYamato University, Katayama, Suita, Osaka. Email: ynarita@eng.hokudai.ac.jp
}

\begin{abstract}
A method is presented for determining the free vibration frequencies of doubly curved, isotropic shallow shells under general edge conditions and is used to obtain accurate natural frequencies for wide range of geometric parameters. Based on the shallow shell theory applicable to thin thickness shells, a method of Ritz is extended to derive a frequency equation wherein the displacement functions are modified to accommodate arbitrary sets of edge conditions for both in-plane and out-of-plane motions. In numerical computation, convergence is tested against series terms and comparison study is made with existing results by other authors. Twenty one sets of frequency parameters are tabulated for a wide range of shell shape and curvature ratio to serve as data for future comparison and practical design purpose.
\end{abstract}

Keywords: Accuracy; free vibration; natural frequency; Ritz, method; shallow shell

\section{Introduction}

Open shallow shell is one of practical shell shapes, and has been widely used as structural components in many mechanical, aeronautical, building and marine structures. These shallow shell components are often subjected to vibration environment and the vibration analysis of shallow shells constitutes important part of structural engineering.

Development in the field of general shell vibration is summarized in a famous monograph [1] compiled by Leissa, and shallow shell vibration is a part of this monograph. The mechanics of shells is described in textbooks, for example in [2], and a handbook on shell vibration was published [3] in 2003. Use of shallow shell structure in automobiles was introduced [4] in connection with composite material.

First notable work on this topic is one [5] by Leissa and Kadi that formulated the exact solution of shallow shells of rectangular planform supported along four edges by shear diaphrams. The shear diaphragm gives the edge condition similar to simple support in the flat plate theory, except that the in-plane displacement parallel to the edge is constrained but displacement perpendicular to the edge is free. For general boundary conditions other than the shear diaphragm, exact solution is not derivable.

By using approximate methods, vibration of cantilevered cylindrical [6] and doubly curved [7] shallow shells were studied in the first half of 1980's. Leissa and one of the present authors analyzed vibration

\footnotetext{
${ }^{\star}$ Corresponding author. Tel.: +62811 4136880

Hokkaido University of Science, Teine, Sapporo Japan
}

of completely free [8] and corner point supported shallow shells [9]. In the 1990's, research on this topic became more active. Effects of edge constraints on natural frequencies were studied [10], and one of these authors published a review paper on shallow shell vibration [11]. $\mathrm{Yu}$ and his co-workers presented free vibration analysis of circular cylindrical shells [12]. Liew and Lim published analysis of shallow shells of curvilinear planform [13], doubly curved shell [14], and use of socalled pb-2 Ritz method on the topic [15]. They also presented a review paper on shallow shell vibration [16].

In the 2000's, Qatu once again summarized on development on shallow shell vibration [17] to follow up his previous review [11]. Design problem was studied [18] for maximizing natural frequencies of laminated shallow shell where more complicated stress-strain relations are included. Monterrubio [19] introduced penalty parameters in the analysis of shallow shell vibration. Qatu studied effect of in-plane edge constraints on frequencies of simply supported doubly curved shallow shells [20].

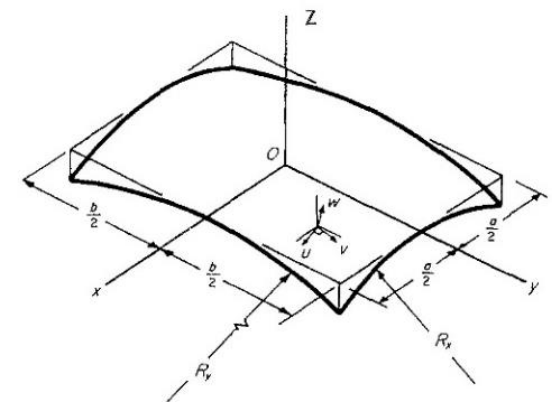

Figure 1. Shallow shell of rectangular planform 
More recently, Mochida et al. [21] and Qatu and Asadi [22] published lists of frequencies for general boundary conditions. Particularly in [21], they extended a method of superposition method to shallow shell vibration for the first time. In the authors' opinion, their numerical results seem most accurate in the literature published this far, but are limited to the case of very thin shell (edge length/ thickness=100). Considering the situations, it is obvious that thorough list of accurate frequency parameters should be tabulated for wide range of general edge conditions, which is the purpose of the present paper.

\section{Analytical Method}

\subsection{Ritz method for general boundary condition}

The quadratic mid-surface of a shallow shell (panel) may be expressed in a rectangular coordinate system as

$$
\phi(x, y)=-\frac{1}{2}\left(\frac{x^{2}}{R_{x}}+2 \frac{x y}{R_{x y}}+\frac{y^{2}}{R_{y}}\right)
$$

where $R_{\mathrm{x}}$ and $R_{\mathrm{y}}$ are the radii of curvature in the $x$ and $y$ directions, respectively, and $R_{\mathrm{xy}}$ is the radius of twist but is not included in this study (i.e., $1 / R_{\mathrm{xy}}=0$ ). For a doubly curved shell, the orientation of the $x-y$ coordinates may be chosen so that $R_{\mathrm{x}}$ and $R_{\mathrm{y}}$ are principal constant curvature radius as shown in Fig.1. The dimension of its planform is given by $a \times b$ and the thickness is $h$. The four sides are subjected to uniform in-plane (i.e., stretching) and out-of-plane (bending) boundary conditions. This shell takes geometric form of a cylindrical shell for " $1 / R_{\mathrm{x}}=$ (finite) and $1 / R_{\mathrm{y}}=0\left(R_{\mathrm{y}}=\infty\right)$ " or " $1 / R_{\mathrm{y}}=$ (finite) and $1 / R_{\mathrm{x}}=0 \quad\left(R_{\mathrm{x}}=\infty\right)$ ". Similarly, it takes form of a spherical shell for $1 / R_{\mathrm{x}}=1 / R_{\mathrm{y}}=$ (finite), and does form of a hyper paraboloidal shell for $1 / R_{\mathrm{x}}=-1 / R_{\mathrm{y}}=$ (finite) where positive curvature exists in $x$ direction and negative curvature in $y$ direction.

Using the Kirchhoff hypothesis, the displacements $u^{*}(x, y, z, t), v^{*}(x, y, z, t)$ and $w^{*}(x, y, z, t)$ of an arbitrary point in a shell are written as

$$
u^{*}=u-z \frac{\partial w}{\partial x}, v^{*}=v-z \frac{\partial w}{\partial y}, w^{*}=w
$$

where $z$ is the coordinate measured from the mid-surface in the direction of outer normal. The $u(x, y, t)$ and $v(x, y, t)$ are displacement components, tangent to the mid-surface and parallel to the $x z$ and $y z$ planes, respectively, and $w(x, y, t)$ is a displacement component normal to the midsurface at a point on the mid-surface.

In the linear theory, the strain components at an arbitrary point $(x, y, z)$ are

$$
\begin{gathered}
\varepsilon_{x}^{*}=\varepsilon_{x}+z \kappa_{x}, \varepsilon_{y}^{*}=\varepsilon_{y}+z \kappa_{y}, \\
\gamma_{x y}^{*}=\gamma_{x y}+z \kappa_{x y}
\end{gathered}
$$

assuming that $z$ is negligible in comparison with $R$, where the membrane strains are given by

$$
\varepsilon_{x}=\frac{\partial u}{\partial x}+\frac{w}{R_{x}}, \varepsilon_{y}=\frac{\partial v}{\partial y}+\frac{w}{R_{y}}, \gamma_{x y}=\frac{\partial v}{\partial x}+\frac{\partial u}{\partial y}
$$

and the curvature changes due to the vibratory displacements are

$$
\kappa_{x}=-\frac{\partial^{2} w}{\partial x^{2}}, \kappa_{y}=-\frac{\partial^{2} w}{\partial y^{2}}, \kappa_{x y}=-2 \frac{\partial^{2} w}{\partial x \partial y}
$$

For an isotropic shallow shell, the stress-strain equation is written as

$$
\left\{\begin{array}{l}
\sigma_{x} \\
\sigma_{y} \\
\tau_{x y}
\end{array}\right\}=\left[\begin{array}{lll}
Q_{11} & Q_{12} & Q_{16} \\
Q_{12} & Q_{22} & Q_{26} \\
Q_{16} & Q_{26} & Q_{66}
\end{array}\right]\left\{\begin{array}{c}
\varepsilon_{x} \\
\varepsilon_{y} \\
\gamma_{x y}
\end{array}\right\}
$$

where the coefficients are elastic constants

$$
Q_{11}=Q_{22}=\frac{E}{1-v^{2}}, Q_{12}=v Q_{11}, Q_{66}=G
$$

where $E$ is the moduli of elasticity, $G=E / 2(1+v)$ is the shear modulus and $v$ is Poisson's ratios.

The force resultants and the moment resultants are obtained by integrating the stresses and the stresses multiplied by $z$, respectively, over the shell thickness $h$, and are written in matrix form as

$$
\left\{\begin{array}{l}
N \\
M
\end{array}\right\}=\left[\begin{array}{ll}
A & B \\
B & D
\end{array}\right]\left\{\begin{array}{l}
\varepsilon \\
\kappa
\end{array}\right\}
$$

where $\{N\},\{M\},\{\varepsilon\}$ and $\{\kappa\}$ are the vectors of force resultants, moment resultants, mid-surface strains and curvatures, respectively, given by

$$
\begin{gathered}
\{N\}=\left\{\begin{array}{l}
N_{x} \\
N_{y} \\
N_{x y}
\end{array}\right\},\{M\}=\left\{\begin{array}{l}
M_{x} \\
M_{y} \\
M_{x y}
\end{array}\right\}, \\
\{\varepsilon\}=\left\{\begin{array}{c}
\varepsilon_{x} \\
\varepsilon_{y} \\
\gamma_{x y}
\end{array}\right\},\{\kappa\}=\left\{\begin{array}{l}
\kappa_{x} \\
\kappa_{y} \\
\kappa_{x y}
\end{array}\right\}
\end{gathered}
$$

and $[A],[B]$ and $[D]$ are the matrices of stiffness coefficients defined by

$$
\begin{aligned}
& {[A]=\left[\begin{array}{lll}
A_{11} & A_{12} & A_{16} \\
A_{12} & A_{22} & A_{26} \\
A_{16} & A_{26} & A_{66}
\end{array}\right]} \\
& {[B]=\left[\begin{array}{lll}
B_{11} & B_{12} & B_{16} \\
B_{12} & B_{22} & B_{26} \\
B_{16} & B_{26} & B_{66}
\end{array}\right]} \\
& {[D]=\left[\begin{array}{lll}
D_{11} & D_{12} & D_{16} \\
D_{12} & D_{22} & D_{26} \\
D_{16} & D_{26} & D_{66}
\end{array}\right]}
\end{aligned}
$$

The stiffness coefficients in Eqs. (10a,b,c) are values determined by 


$$
\begin{gathered}
A_{i j}=h Q_{i j}, B_{i j}=0 \\
D_{i j}=\left(\frac{h}{12}\right) Q_{i j}
\end{gathered}
$$

$(i, j=1,2,6)$ for specific case of shallow shells composed of isotropic material. For more complicated shells including laminated composite materials, Eqs. (11) take more complicated form as shown in Ref. [18].

In the present study, the free vibration problem can be solved by means of the Ritz method. This requires the evaluation of energy functionals. The strain energy stored in a shell during elastic deformation is written in the classical (thin) shallow shell theory by

$$
V=V_{s}+V_{b s}+V_{b}
$$

where $V_{s}, V_{b s}$ and $V_{b}$ are the parts of the total strain energy due to stretching, bending- stretching coupling and bending, respectively:

$$
\begin{gathered}
V_{s}=\frac{1}{2} \iint\{\varepsilon\}^{T}[A]\{\varepsilon\} \text { dArea } \\
V_{b s}=\frac{1}{2} \iint\left(\{\kappa\}^{T}[B]\{\varepsilon\}+\{\varepsilon\}^{T}[B]\{\kappa\}\right) \text { dArea } \\
V_{b}=\frac{1}{2} \iint\{\kappa\}^{T}[D]\{\kappa\} \text { dArea }
\end{gathered}
$$

The kinetic energy of the panel due to translational motion only is given by

$$
T=\frac{1}{2} \rho h \iint\left[\left(\frac{\partial u}{\partial t}\right)^{2}+\left(\frac{\partial v}{\partial t}\right)^{2}+\left(\frac{\partial w}{\partial t}\right)^{2}\right] d \text { Area }
$$

where $\rho$ is the mass density of the shell per unit volume.

For simplicity in the formulation, the following dimensionless quantities are introduced.

$$
\begin{aligned}
& \xi=\frac{2 x}{a}, \eta=\frac{2 y}{b} \text { (dimensionless coordinates) } \\
& \Omega=\omega a^{2} \sqrt{\frac{\rho h}{D}} \text { (dimensionless frequency parameter) } \\
& D=\frac{E h^{3}}{12\left(1-v^{2}\right)} \text { (reference plate stiffness) }
\end{aligned}
$$

In the Ritz method the displacements may be assumed in the form

$$
\begin{aligned}
& u(\xi, \eta, t)=\sum_{i=0}^{M-1} \sum_{j=0}^{N-1} P_{i j} X_{i}(\xi) Y_{j}(\eta) \sin \omega t \\
& v(\xi, \eta, t)=\sum_{k=0}^{M-1} \sum_{l=0}^{N-1} Q_{k l} X_{k}(\xi) Y_{l}(\eta) \sin \omega t
\end{aligned}
$$

$$
w(\xi, \eta, t)=\sum_{m=0}^{M-1} \sum_{n=0}^{N-1} R_{m n} X_{m}(\xi) Y_{n}(\eta) \sin \omega t(16 \mathrm{c})
$$

where $P_{i j}, Q_{k l}$ and $R_{m n}$ are unknown coefficients and $X_{i}(\xi), Y_{j}(\eta), .$. and $Y_{n}(\eta)$ are the functions that satisfy at least the kinematical boundary conditions at the edges. The upper limit in each of the summations (16) is arbitrary but is unified here for simplicity in the convergence test.

After substituting Eqs.(16) into the functional $L$

$$
L=T_{\max }-V_{\max }
$$

composed of the maximum strain and kinetic energies obtained from Eqs.(12) and (14), the stationary value is obtained by

$$
\frac{\partial L}{\partial P_{i j}}=0, \frac{\partial L}{\partial Q_{k l}}=0, \frac{\partial L}{\partial R_{m n}}=0
$$

$(18 \mathrm{a}, \mathrm{b}, \mathrm{c})$

$(i, k, m=0,1,2, . .,(M-1) ; j, l, n=0,1,2, \ldots,(N-1))$

The result of the minimization process (18) yields a set of homogeneous, linear simultaneous equations in the unknowns $\left\{P_{i j}, Q_{k l}, R_{m n}\right\}$. For non-trivial solutions, the determinant of the coefficient matrix is set to zero. The $(M \times N) \times 3$ eigenvalues may be extracted and the lowest several eigenvalues (natural frequencies) are important from a practical viewpoint.

The above procedure is a standard routine of the Ritz method, and is modified to incorporate arbitrary edge conditions. This approach introduces the following polynomials

$$
\begin{aligned}
& X_{i}(\xi)=\xi^{i}(1+\xi)^{B u 1}(1-\xi)^{B u 3} \\
& Y_{j}(\eta)=\eta^{j}(1+\eta)^{B u 2}(1-\eta)^{B u 4} \\
& X_{k}(\xi)=\xi^{k}(1+\xi)^{B v 1}(1-\xi)^{B v 3} \\
& Y_{l}(\eta)=\eta^{l}(1+\eta)^{B v 2}(1-\eta)^{B v 4} \\
& X_{m}(\xi)=\xi^{m}(1+\xi)^{B w 1}(1-\xi)^{B w 3} \\
& Y_{n}(\eta)=\eta^{n}(1+\eta)^{B w 2}(1-\eta)^{B w 4}
\end{aligned}
$$

where $B_{r s} \quad(r=u, v, w ; s=1,2,3,4)$ is the boundary index [18] which is used to satisfy the kinematic boundary conditions. The capital letter $B$ stands for Boundary. The first subscript letter in $B_{r s}$ indicates which displacement $(u, v$ or $w)$ is dealt with and the second subscript number indicates which edge, Edge (1),.. or Edge (4), is under consideration. The Edge (1),(2),(3) and (4) denote the boundary along $x=-a / 2, y=-$ $b / 2, x=a / 2$ and $y=b / 2$, respectively (See Fig. 1). 
For in-plane displacements $u$ and $v, B_{r s}=0(r=u, v$; $s=1,2,3,4)$ denote that the specified displacement along the specified edge is free and $B_{r s}=1$ denote that the displacement is rigidly fixed. For out-of-plane displacement $w, B_{r s}=0,1$ and $2(r=w ; s=1,2,3,4)$ denote that the specified displacement along the specified edge is free, simply supported and clamped, respectively. With such boundary indices, one can accommodate arbitrary sets of both in-plane and out-of-plane boundary conditions in the vibration analysis and computation.

The introduction of the boundary index makes it possible to deal with a tremendous number of edge conditions in the analysis. The number of combinations in the boundary condition is $(2 \times 2 \times 3)^{4}=20736$, when one of the two conditions (free or fixed) in $u$ and $v$ and one of the three conditions (free, simply supported or clamped) in $w$ are imposed along each of the four edges. This is significantly larger than the plate analysis where only out-of-plane displacement is concerned. The present vibration analysis can calculate natural frequencies for any of these combinations.

In the following numerical examples, however, the boundary conditions are limited to those similar to the standard plate boundary conditions, such as free edge, simply supported edge and clamped edge. For example, at the Edge (1) along $x=-a / 2$

$$
\begin{aligned}
B_{\mathrm{u} 1}= & B_{\mathrm{v} 1}=B_{\mathrm{w} 1}=0 \text { for the free edge (no constraint), } \\
& \text { denoted by } \mathrm{F} \\
B_{\mathrm{u} 1}= & 0, B_{\mathrm{v} 1}=1, B_{\mathrm{w} 1}=1 \text { for the simply supported edge } \\
& \text { denoted by } \mathrm{S} \\
B_{\mathrm{u} 1}= & B_{\mathrm{v} 1}=1, B_{\mathrm{w} 1}=2 \text { for the clamped edge } \\
& \text { denoted by C }
\end{aligned}
$$

The entire set of boundary conditions is denoted by four capital letter, such as CSFF, in the counterclockwise starting from Edge(1).

\subsection{Exact solution for specific boundary condition}

When a shallow shell of rectangular planform is supported along four edges by shear diaphragms, the exact solution is possible [1][3][5] by assuming

$$
\begin{aligned}
& u(x, y, t)=P_{i j} \cos \frac{i \pi x}{a} \sin \frac{j \pi y}{b} \sin \omega t \\
& v(x, y, t)=Q_{k l} \sin \frac{k \pi x}{a} \cos \frac{l \pi y}{b} \sin \omega t \\
& w(x, y, t)=R_{m n} \sin \frac{m \pi x}{a} \sin \frac{n \pi y}{b} \sin \omega t
\end{aligned}
$$

Equations (21) are substituted in the governing equation, and the exact solution is obtained in the form of $3 \times 3$ frequency matrix. This solution procedure is given in

\begin{tabular}{|c|c|c|c|c|c|c|}
\hline & $\Omega_{1}$ & $\Omega_{2}$ & $\Omega_{3}$ & $\Omega_{4}$ & $\Omega_{5}$ & $\Omega_{6}$ \\
\hline \multicolumn{7}{|c|}{ Spherical shell $(R x / R y=1, a / R x=0.2)$} \\
\hline $8 \times 8$ & 23.715 & 51.052 & 51.052 & 80.021 & 99.563 & 99.563 \\
\hline $10 \times 10$ & 23.715 & 51.052 & 51.052 & 80.021 & 99.543 & 99.543 \\
\hline $12 \times 12$ & 23.715 & 51.052 & 51.052 & 80.021 & 99.542 & 99.542 \\
\hline Exact & 23.715 & 51.052 & 51.052 & 80.021 & 99.542 & 99.542 \\
\hline Ref.[22] & 23.70 & 51.04 & 51.04 & 80.02 & - & - \\
\hline \multicolumn{7}{|c|}{ Spherical shell $(R x / R y=1, a / R x=0.5)$} \\
\hline $8 \times 8$ & 38.080 & 59.134 & 59.134 & 85.361 & 103.88 & 103.88 \\
\hline $10 \times 10$ & 38.080 & 59.134 & 59.134 & 85.361 & 103.85 & 103.85 \\
\hline $12 \times 12$ & 38.080 & 59.134 & 59.134 & 85.361 & 103.85 & 103.85 \\
\hline Exact & 38.080 & 59.134 & 59.134 & 85.361 & 103.85 & 103.85 \\
\hline Ref.[22] & 38.01 & 59.10 & 59.10 & 85.32 & - & - \\
\hline \multicolumn{7}{|c|}{ Cylindrical shell $(R x / R y=0, a / R x=0.2)$} \\
\hline $8 \times 8$ & 20.786 & 49.391 & 50.451 & 79.203 & 98.700 & 99.420 \\
\hline $10 \times 10$ & 20.786 & 49.391 & 50.451 & 79.203 & 98.680 & 99.400 \\
\hline $12 \times 12$ & 20.786 & 49.391 & 50.451 & 79.203 & 98.680 & 99.400 \\
\hline Exact & 20.786 & 49.391 & 50.451 & 79.203 & 98.680 & 99.400 \\
\hline Ref.[22] & 20.78 & 49.39 & 50.44 & 79.19 & - & - \\
\hline \multicolumn{7}{|c|}{ Cylindrical shell $(R x / R y=0, a / R x=0.5)$} \\
\hline $8 \times 8$ & 25.509 & 49.613 & 55.861 & 80.479 & 98.616 & 103.03 \\
\hline $10 \times 10$ & 25.509 & 49.613 & 55.861 & 80.479 & 98.616 & 103.03 \\
\hline $12 \times 12$ & 25.509 & 49.612 & 55.861 & 80.479 & 98.593 & 103.01 \\
\hline Exact & 25.509 & 49.612 & 55.861 & 80.479 & 98.594 & 103.01 \\
\hline Ref.[22] & 25.48 & 49.61 & 55.84 & 80.46 & - & - \\
\hline \multicolumn{7}{|c|}{ Hyperbolic paraboloidal shell $(R x / R y=-1, a / R x=0.2)$} \\
\hline $8 \times 8$ & 19.659 & 49.924 & 49.924 & 78.873 & 99.244 & 99.244 \\
\hline $10 \times 10$ & 19.659 & 49.924 & 49.924 & 78.873 & 99.224 & 99.224 \\
\hline $12 \times 12$ & 19.659 & 49.924 & 49.924 & 78.873 & 99.224 & 99.224 \\
\hline Exact & 19.659 & 49.924 & 49.924 & 78.873 & 99.224 & 99.224 \\
\hline Ref.[22] & 19.66 & 49.92 & 49.92 & 78.85 & - & - \\
\hline \multicolumn{7}{|c|}{ Hyperbolic paraboloidal shell $(\mathrm{Rx} / \mathrm{Ry}=-1, \mathrm{a} / \mathrm{Rx}=0.5)$} \\
\hline $8 \times 8$ & 19.252 & 52.806 & 52.806 & 78.438 & 101.96 & 101.96 \\
\hline $10 \times 10$ & 19.252 & 52.805 & 52.805 & 78.438 & 101.94 & 101.94 \\
\hline $12 \times 12$ & 19.252 & 52.805 & 52.805 & 78.438 & 101.94 & 101.94 \\
\hline Exact & 19.252 & 52.805 & 52.805 & 78.438 & 101.94 & 101.94 \\
\hline Ref.[22] & 19.25 & 52.79 & 52.79 & 78.46 & - & - \\
\hline
\end{tabular}
detail in Refs. [3][5].

\section{Numerical Examples and Accuracy of Solution}

\subsection{Convergence and comparison of the solution}

In numerical examples, square planform $(a / b=1)$ and moderately thin thickness $(a / h=20)$ are used. Poisson's ratio is kept as $v=0.3$.
Table 1 Convergence and comparison of frequency parameters $\Omega$ of simply supported shallow shells (SSSS), $a / b=1, a / h=20, v=0.3$.

Table 1 presents convergence study of frequency parameters of spherical $\left(R_{\mathrm{x}} / R_{\mathrm{y}}=1\right)$, cylindrical $\left(R_{\mathrm{x}} / R_{\mathrm{y}}=0\right)$ and hyperbolic paraboloidal $\left(R_{\mathrm{x}} / R_{\mathrm{y}}=-1\right)$ shells of square planform. These shells (SSSS) are supported by shear diaphram along four edges. For each shell configuration, two degrees of curvature $a / R_{\mathrm{x}}=0.2$ and 0.5 are used. The present results are calculated for the number of terms $8 \times 8$, $10 \times 10$ and $12 \times 12$ for each of $u, v$ and $w$ that yield frequency matrix size of $192 \times 192,300 \times 300$ and $432 \times 432$, respectively.

The present parameters converge well within five significant figures, and show exact match with the exact results obtained from Eq. (21). They are also compared to the parameters in Ref. [22], and are generally in good agreement with slight differences. In the following tables, the present results are calculated by using the $12 \times 12$ solution and presented in five significant figures. 
Table 2 Comparison of frequency parameters $\Omega$ of shallow shells, $a / R x=0.5, a / b=1, a / h=100, v=0.3$.

\begin{tabular}{|c|c|c|c|c|c|c|}
\hline & $\Omega_{1}$ & $\Omega_{2}$ & $\Omega_{3}$ & $\Omega_{4}$ & $\Omega_{5}$ & $\Omega_{6}$ \\
\hline \multicolumn{7}{|l|}{ ccss } \\
\hline \multicolumn{7}{|c|}{ Spherical shell $(R x / R y=1)$} \\
\hline Present & 171.60 & 180.27 & 186.99 & 195.32 & 204.04 & 210.09 \\
\hline Ref.[21] & 171.6 & 180.3 & 187.0 & 195.3 & 204.1 & 210.1 \\
\hline \multicolumn{7}{|c|}{ Cylindrical shell $(\mathrm{Rx} / \mathrm{Ry}=0)$} \\
\hline Present & 72.375 & 105.48 & 127.24 & 133.02 & 147.49 & 168.62 \\
\hline Ref.[21] & 72.37 & 105.5 & 127.2 & 133.0 & 147.5 & 168.6 \\
\hline \multicolumn{7}{|c|}{ Hyperbolic paraboloidal shell $(R x / R y=-1)$} \\
\hline Present & 94.181 & 122.38 & 136.98 & 149.24 & 173.01 & 173.62 \\
\hline Ref.[21] & 94.18 & 122.4 & 137.0 & 149.2 & 173.0 & 173.6 \\
\hline \multicolumn{7}{|l|}{$\operatorname{cscc}$} \\
\hline \multicolumn{7}{|c|}{ Spherical shell $(R x / R y=1)$} \\
\hline Present & 179.90 & 187.88 & 192.50 & 202.47 & 208.02 & 228.73 \\
\hline Ref.[21] & 179.9 & 187.9 & 192.5 & 202.5 & 208.0 & 228.7 \\
\hline \multicolumn{7}{|c|}{ Cylindrical shell $(\mathrm{Rx} / \mathrm{Ry}=0)$} \\
\hline Present & 95.798 & 116.43 & 145.24 & 150.67 & 170.30 & 187.31 \\
\hline Ref.[21] & 95.81 & 116.4 & 145.2 & 150.6 & 170.3 & 187.3 \\
\hline \multicolumn{7}{|c|}{ Hyperbolic paraboloidal shell $(\mathrm{Rx} / \mathrm{Ry}=-1)$} \\
\hline Present & 131.20 & 139.90 & 154.35 & 158.42 & 186.02 & 189.83 \\
\hline Ref.[21] & 131.2 & 139.9 & 154.4 & 158.4 & 186.0 & 189.8 \\
\hline \multicolumn{7}{|l|}{$\operatorname{cccc}$} \\
\hline \multicolumn{7}{|c|}{ Spherical shell $(R x / R y=1)$} \\
\hline Present & 191.99 & 191.99 & 196.93 & 209.96 & 216.19 & 242.22 \\
\hline Ref.[21] & 192.0 & 192.0 & 169.9 & 210.0 & 216.2 & 242.2 \\
\hline \multicolumn{7}{|c|}{ Cylindrical shell $(\mathrm{Rx} / \mathrm{Ry}=0)$} \\
\hline Present & 99.263 & 119.00 & 151.13 & 156.35 & 172.52 & 192.43 \\
\hline Ref.[21] & 99.26 & 119.0 & 151.1 & 156.3 & 172.5 & 192.4 \\
\hline \multicolumn{7}{|c|}{ Hyperbolic paraboloidal shell $(\mathrm{Rx} / \mathrm{Ry}=-1)$} \\
\hline Present & 157.35 & 157.35 & 157.41 & 166.52 & 204.03 & 208.69 \\
\hline Ref.[21] & 157.3 & 157.3 & 157.4 & 166.5 & 204.0 & 208.7 \\
\hline
\end{tabular}

Table 2 is another comparison study with values of Ref. [21] by Mochida and his co-workers. Note that only in Table 2, the thickness ratio is kept as $a / h=100$ (very thin) due to the need for comparison, while in all other tables, the ratio is $a / h=20$ (moderately thin) throughout. They [21] extended the method of superposition, commonly used in plate vibration analysis, to shallow shell vibration analysis. Three sets of boundary condition CCSS, CSCC and CCCC are considered. When the present values are rounded with four significant figures, most of the results are exactly identical with the referred values [21] for wide ranges of boundary conditions, shell configuration and degree of curvature. Thus, the accuracy of the present solution is well demonstrated

\subsection{Comprehensive results of shallow shells}

Table 3(a) presents the lowest six frequency parameters $\Omega$ of shallow spherical shells $\left(R_{\mathrm{x}} / R_{\mathrm{y}}=1\right)$ of square planform $(a / b=1)$ with moderate thickness $(a / h=20)$ for 21 different sets of boundary conditions. The degree of curvature is taken as $a / R=0.2$. Table $3(\mathrm{~b})$ is the same format as Table 3(a) except that the curvature is larger in $a / R=0.5$.
Table 3(a) Frequency parameters $\Omega$ of shallow spherical shells, $R_{\mathrm{x}} / R_{\mathrm{y}}=1, a / R_{\mathrm{x}}=a / R_{\mathrm{y}}=0.2, a / b=1, a / h=20, v=0.3$.

\begin{tabular}{ccccccc}
\hline B.C. & $\Omega_{1}$ & $\Omega_{2}$ & $\Omega_{3}$ & $\Omega_{4}$ & $\Omega_{5}$ & $\Omega_{6}$ \\
\hline FFFF & 13.460 & 19.563 & 25.991 & 34.838 & 34.838 & 61.770 \\
SFFF & 6.6294 & 15.343 & 25.400 & 27.084 & 49.196 & 51.604 \\
CFFF & 3.7518 & 8.4865 & 21.518 & 28.262 & 30.515 & 44.098 \\
SSFF & 3.3668 & 17.321 & 20.788 & 39.706 & 51.532 & 54.319 \\
CSFF & 5.5638 & 19.350 & 25.461 & 44.633 & 53.198 & 54.137 \\
CCFF & 7.8923 & 23.895 & 27.922 & 49.256 & 62.962 & 66.231 \\
SFSF & 10.066 & 16.105 & 38.895 & 39.592 & 46.867 & 71.588 \\
CFSF & 15.629 & 21.677 & 41.802 & 49.902 & 56.556 & 78.415 \\
SSSF & 12.221 & 30.508 & 41.852 & 60.149 & 63.216 & 64.384 \\
CSSF & 17.921 & 33.738 & 51.986 & 65.351 & 68.576 & 101.91 \\
CCSF & 18.464 & 38.646 & 52.338 & 72.133 & 75.507 & 106.00 \\
CFCF & 25.385 & 28.722 & 45.607 & 61.561 & 67.417 & 80.888 \\
SCSF & 12.951 & 35.762 & 42.269 & 64.111 & 64.384 & 73.590 \\
CSCF & 26.453 & 38.140 & 63.338 & 68.064 & 78.336 & 109.64 \\
CCCF & 26.907 & 42.737 & 63.637 & 77.905 & 81.563 & 117.42 \\
SSSS & 23.715 & 51.052 & 51.052 & 80.021 & 99.542 & 99.542 \\
CSSS & 27.338 & 53.342 & 60.177 & 87.149 & 101.11 & 113.97 \\
CCSS & 30.659 & 62.026 & 62.352 & 93.819 & 115.29 & 115.45 \\
CSCS & 32.577 & 56.377 & 70.634 & 95.546 & 103.05 & 128.77 \\
CCCS & 35.610 & 64.875 & 72.385 & 101.74 & 117.10 & 131.04 \\
CCCC & 40.422 & 74.713 & 74.713 & 109.14 & 132.24 & 133.06
\end{tabular}

Table3(b) Frequency parameters $\Omega$ of shallow spherical shells, $R_{\mathrm{x}} / R_{\mathrm{y}}=1, a / R_{\mathrm{x}}=a / R_{\mathrm{y}}=0.5, a / b=1, a / h=20, v=0.3$.

\begin{tabular}{ccccccc}
\hline B.C. & $\Omega_{1}$ & $\Omega_{2}$ & $\Omega_{3}$ & $\Omega_{4}$ & $\Omega_{5}$ & $\Omega_{6}$ \\
\hline FFFF & 13.414 & 19.392 & 32.522 & 34.952 & 34.952 & 64.993 \\
SFFF & 6.5501 & 16.251 & 25.460 & 31.034 & 52.846 & 57.290 \\
CFFF & 4.7227 & 8.3836 & 22.089 & 28.777 & 32.298 & 46.445 \\
SSFF & 3.3328 & 17.287 & 24.642 & 48.041 & 53.468 & 58.038 \\
CSFF & 6.3859 & 19.708 & 27.976 & 52.485 & 54.138 & 56.256 \\
CCFF & 11.139 & 23.818 & 32.726 & 57.426 & 63.892 & 69.438 \\
SFSF & 11.118 & 15.910 & 42.201 & 47.454 & 48.857 & 76.185 \\
CFSF & 17.086 & 26.233 & 50.914 & 52.202 & 57.953 & 83.723 \\
SSSF & 13.137 & 42.426 & 44.253 & 64.383 & 66.009 & 69.874 \\
CSSF & 21.832 & 45.242 & 54.358 & 71.892 & 74.084 & 105.86 \\
CCSF & 22.097 & 50.022 & 54.536 & 77.648 & 81.379 & 107.00 \\
CFCF & 37.260 & 38.449 & 55.161 & 63.247 & 68.605 & 86.273 \\
SCSF & 13.672 & 44.410 & 47.411 & 64.384 & 69.935 & 79.514 \\
CSCF & 37.802 & 49.827 & 65.270 & 74.501 & 83.417 & 113.60 \\
CCCF & 37.993 & 55.089 & 65.419 & 83.845 & 86.719 & 121.32 \\
SSSS & 38.080 & 59.134 & 59.134 & 85.361 & 103.85 & 103.85 \\
CSSS & 41.361 & 61.292 & 67.571 & 92.265 & 105.39 & 117.76 \\
CCSS & 44.688 & 69.242 & 69.928 & 98.789 & 119.07 & 119.22 \\
CSCS & 46.965 & 64.210 & 77.040 & 100.41 & 107.29 & 128.77 \\
CCCS & 50.623 & 72.403 & 78.805 & 106.52 & 120.86 & 134.53 \\
CCCC & 58.167 & 81.187 & 81.187 & 113.82 & 135.61 & 137.50 \\
\hline & & & & & & \\
\hline
\end{tabular}


Table 4(a) Frequency parameters $\Omega$ of shallow cylindrical shells, $R_{\mathrm{x}}=$ (infinity) $, a / R_{\mathrm{y}}=0.2, a / b=1, a / h=20, v=0.3$.

\begin{tabular}{ccccccc}
\hline B.C. & $\Omega_{1}$ & $\Omega_{2}$ & $\Omega_{3}$ & $\Omega_{4}$ & $\Omega_{5}$ & $\Omega_{6}$ \\
\hline FFFF & 13.463 & 20.120 & 24.765 & 34.783 & 34.906 & 61.080 \\
SFFF & 6.6433 & 15.762 & 25.444 & 26.011 & 48.826 & 51.057 \\
CFFF & 3.8031 & 8.520 & 21.967 & 27.283 & 31.040 & 43.507 \\
SSFF & 3.3642 & 17.606 & 19.790 & 38.566 & 51.306 & 53.876 \\
CSFF & 5.6203 & 19.155 & 25.344 & 43.415 & 52.701 & 54.069 \\
CCFF & 7.2165 & 24.291 & 27.072 & 48.195 & 62.925 & 65.837 \\
SFSF & 10.293 & 16.180 & 36.802 & 39.703 & 46.889 & 70.965 \\
CFSF & 15.820 & 20.652 & 39.843 & 50.076 & 56.427 & 77.325 \\
SSSF & 12.306 & 28.033 & 41.887 & 59.537 & 61.866 & 64.383 \\
CSSF & 17.374 & 31.420 & 51.973 & 64.044 & 67.958 & 101.23 \\
CCSF & 18.147 & 36.578 & 52.369 & 71.598 & 74.409 & 106.18 \\
CFCF & 22.728 & 26.490 & 43.729 & 61.700 & 67.313 & 79.829 \\
SCSF & 13.345 & 33.608 & 42.359 & 63.602 & 64.383 & 72.471 \\
CSCF & 23.879 & 35.887 & 63.362 & 66.802 & 77.744 & 108.98 \\
CCCF & 24.466 & 40.558 & 63.695 & 76.806 & 81.039 & 116.81 \\
SSSS & 20.786 & 49.391 & 50.451 & 79.203 & 98.680 & 99.400 \\
CSSS & 24.612 & 51.746 & 59.574 & 86.374 & 100.26 & 113.84 \\
CCSS & 28.366 & 60.815 & 61.677 & 93.135 & 114.62 & 115.26 \\
CSCS & 29.790 & 54.843 & 70.118 & 94.807 & 102.22 & 128.77 \\
CCCS & 32.959 & 63.515 & 71.936 & 101.07 & 116.37 & 130.89 \\
CCCC & 37.829 & 73.541 & 74.364 & 108.53 & 131.81 & 132.63
\end{tabular}

Table 4(b) Frequency parameters $\Omega$ of shallow cylindrical shells, $R_{\mathrm{x}}=$ (infinity), $a / R_{\mathrm{y}}=0.5, a / b=1, a / h=20, v=0.3$.

\begin{tabular}{|c|c|c|c|c|c|c|}
\hline B.C. & 1 & 2 & 3 & 4 & $?_{5}$ & 6 \\
\hline$F F$ & .439 & 21.283 & 28.119 & 34.685 & 35.450 & 0 \\
\hline EF & 5 & 7 & 0 & & 39 & 147 \\
\hline$F F$ & 6 & 3 & 14 & & 37 & 520 \\
\hline & & & & & & 461 \\
\hline CSFF & 9 & 1 & & & & 5 \\
\hline CCFF & 8.4374 & 25.562 & & 51 & 47 & 845 \\
\hline SFSF & 13.103 & 16.415 & 37. & 43 & 66 & 72.235 \\
\hline C & 18.606 & 21.000 & 40 & 53 & 83 & 77.330 \\
\hline 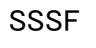 & 1 & 0 & & 3 & & 6 \\
\hline CSSF & & & & & & \\
\hline C & 20 & 3 & & & & 09 \\
\hline CFCF & 25 & 26 & & & & 395 \\
\hline SCSF & 15.790 & 36.420 & 45 & 64 & 66 & 72.867 \\
\hline CSCF & 26.034 & 37.687 & 65.6 & 67 & 79 & 109.57 \\
\hline CCCF & 26.625 & & & & & \\
\hline SSSS & 25.509 & 49.612 & 55.8 & & & 103.01 \\
\hline CSSS & 29.072 & 52. & 64 & 87 & 10 & 116.97 \\
\hline $\operatorname{ccs}$ & 34 & 6 & 66 & 9 & 11 & 118.46 \\
\hline Cs & 33 & 55 & & & 21 & .77 \\
\hline $\mathrm{C}$ & 38.182 & 64.5 & 76.2 & 102.52 & .41 & 133.67 \\
\hline $\operatorname{cccc}$ & 46.241 & 74.300 & 79.239 & 110.14 & 132.35 & 135.51 \\
\hline
\end{tabular}

Table 5(a) Frequency parameters $\Omega$ of shallow cylindrical shells, $R_{\mathrm{y}}=$ (infinity), $a / R_{\mathrm{x}}=0.2, a / b=1, a / h=20, v=0.3$.

\begin{tabular}{lllllll}
\hline B.C. & $\Omega_{1}$ & $\Omega_{2}$ & $\Omega_{3}$ & $\Omega_{4}$ & $\Omega_{5}$ & $\Omega_{6}$ \\
\hline$\underline{\text { FFFF }}$ & $\underline{13.463}$ & $\underline{20.120}$ & $\underline{24.765}$ & $\underline{34.783}$ & $\underline{34.906}$ & $\underline{61.080}$ \\
SFFF & 6.6292 & 14.925 & 25.340 & 26.889 & 48.649 & 50.799 \\
CFFF & 3.4675 & 8.4672 & 21.301 & 27.977 & 30.489 & 44.084 \\
$\underline{\text { SSFF }}$ & $\underline{3.3642}$ & $\underline{17.606}$ & $\underline{19.790}$ & $\underline{38.566}$ & $\underline{51.306}$ & $\underline{53.876}$ \\
CSFF & 5.3411 & 19.816 & 24.606 & 43.658 & 53.213 & 54.191 \\
$\underline{\text { CCFF }}$ & $\underline{7.2160}$ & $\underline{24.291}$ & $\underline{27.072}$ & $\underline{48.195}$ & $\underline{62.925}$ & $\underline{65.837}$ \\
SFSF & 9.6216 & 16.062 & 38.095 & 38.927 & 46.707 & 70.902 \\
CFSF & 15.125 & 21.586 & 41.173 & 49.350 & 56.387 & 78.225 \\
SSSF & 11.656 & 29.019 & 41.175 & 59.160 & 62.868 & 64.384 \\
CSSF & 17.291 & 32.612 & 51.402 & 65.052 & 67.756 & 101.44 \\
CCSF & 18.233 & 37.348 & 51.833 & 71.290 & 75.213 & 105.65 \\
CFCF & 24.782 & 28.631 & 45.173 & 61.099 & 67.253 & 80.746 \\
SCSF & 13.046 & 34.169 & 41.694 & 63.122 & 64.384 & 73.255 \\
CSCF & 25.874 & 37.417 & 62.847 & 67.846 & 77.615 & 109.24 \\
CCCF & 26.364 & 41.684 & 63.207 & 77.660 & 80.811 & 117.00 \\
$\underline{\text { SSSS }}$ & $\underline{20.786}$ & $\underline{49.391}$ & $\underline{50.451}$ & $\underline{79.203}$ & $\underline{98.680}$ & $\underline{99.400}$ \\
CSSS & 25.089 & 52.840 & 58.803 & 86.447 & 100.98 & 113.22 \\
$\underline{\text { CCSS }}$ & $\underline{28.366}$ & $\underline{60.815}$ & $\underline{61.677}$ & $\underline{93.135}$ & $\underline{114.62}$ & $\underline{115.26}$ \\
$\underline{\text { CSCS }}$ & 31.159 & 56.022 & 69.447 & 94.935 & 102.95 & 128.77 \\
CCCS & 33.866 & 64.448 & 71.213 & 101.12 & 117.01 & 130.41 \\
$\underline{\text { CCCC }}$ & $\underline{37.829}$ & $\underline{73.541}$ & $\underline{74.364}$ & $\underline{108.53}$ & $\underline{131.81}$ & $\underline{132.63}$ \\
\hline
\end{tabular}

Addition of curvature causes frequencies to be increased. In Table 3(a), the average increase from flat plates for the fundamental frequencies in $\Omega_{1}$ is 9 percent, including the highest increase 20 percent of SSSS shell. The deeper curvature $a / R=0.5$ in Table $3(\mathrm{~b})$ shows the average increase of 39 percent in $\Omega_{1}$ with the maximum 93 percent of SSSS shell.

Table 4(a) and (b) list up the lowest six frequency parameters of shallow cylindrical shells with $R_{\mathrm{x}}=$ (infinity) and $a / R_{\mathrm{y}}=0.2$ and $a / R_{\mathrm{y}}=0.5$, respectively. This represents straight edges of the shell along the $x$ axis, and curvature is given only in $y$ direction. When the increase of the fundamental frequencies due to the curvature increase is considered, the average percent increases are 4 percent and 20 percent in Table 4(a) and (b), respectively. Roughly speaking, this effect is a half of the spherical shells, and the effect of curvature increase in one direction is a half of curvatures in two directions of spherical shells.

Tables 5(a) and (b) also tabulate the lowest six frequency parameters of shallow cylindrical shells, but with $R_{\mathrm{y}}=$ (infinity) and $a / R_{\mathrm{x}}=0.2$ and $a / R_{\mathrm{x}}=0.5$, respectively. Straight edges of the shell exist along the $y$ axis, and curvature is only in $x$ direction. For cylindrical shells with FFFF, SSSS and CCCC, the results are the identical as in Table 4(a) and (b) due to uniform boundary condition along four edges, and also shells with SSFF, CCFF and CCSS give the identical results as in Table 4(a) and (b) since the 90 degree rotation (or flipping about a diagonal symmetric axis) of the shell gives essentially the same boundary conditions. 
Table 5(b) Frequency parameters $\Omega$ of shallow cylindrical shells, $R_{\mathrm{y}}=$ (infinity), $a / R_{\mathrm{x}}=0.5, a / b=1, a / h=20, v=0.3$.

\begin{tabular}{lllllll}
\hline B.C. & $\Omega_{1}$ & $\Omega_{2}$ & $\Omega_{3}$ & $\Omega_{4}$ & $\Omega_{5}$ & $\Omega_{6}$ \\
\hline$\underline{\text { FFFF }}$ & $\underline{13.439}$ & $\underline{21.284}$ & $\underline{28.119}$ & $\underline{34.685}$ & $\underline{35.450}$ & $\underline{61.010}$ \\
SFFF & 6.5537 & 14.962 & 25.159 & 30.710 & 49.135 & 52.689 \\
CFFF & 3.4369 & 8.2686 & 21.129 & 28.647 & 31.417 & 46.386 \\
$\underline{\text { SSFF }}$ & $\underline{3.3474}$ & $\underline{17.927}$ & $\underline{22.681}$ & $\underline{40.569}$ & $\underline{51.774}$ & $\underline{56.461}$ \\
CSFF & 5.2853 & 22.217 & 24.740 & 46.690 & 53.805 & 56.634 \\
$\underline{\text { CCFF }}$ & $\underline{8.4374}$ & $\underline{25.562}$ & $\underline{29.549}$ & $\underline{51.099}$ & $\underline{63.347}$ & $\underline{67.845}$ \\
SFSF & 9.5542 & 15.696 & 38.833 & 44.561 & 46.547 & 71.739 \\
CFSF & 14.777 & 25.870 & 47.889 & 48.900 & 57.008 & 80.148 \\
SSSF & 11.506 & 34.831 & 41.059 & 59.650 & 64.384 & 67.893 \\
CSSF & 19.550 & 39.366 & 51.429 & 68.895 & 70.218 & 103.10 \\
CCSF & 21.313 & 43.499 & 51.948 & 72.439 & 79.696 & 105.04 \\
CFCF & 35.338 & 38.115 & 52.645 & 60.699 & 67.650 & 85.438 \\
SCSF & 14.725 & 39.385 & 41.653 & 63.678 & 64.384 & 77.594 \\
CSCF & 36.143 & 45.846 & 62.698 & 73.258 & 78.852 & 111.13 \\
CCCF & 36.473 & 49.547 & 63.134 & 82.043 & 82.448 & 118.79 \\
$\underline{\text { SSSS }}$ & $\underline{25.509}$ & $\underline{49.612}$ & $\underline{55.861}$ & $\underline{80.479}$ & $\underline{98.594}$ & $\underline{103.01}$ \\
$\underline{\text { CSSS }}$ & 31.345 & 58.522 & 59.672 & 88.079 & 104.65 & 113.21 \\
$\underline{\text { CCSS }}$ & $\underline{34.217}$ & $\underline{61.768}$ & $\underline{66.606}$ & $\underline{94.692}$ & $\underline{114.64}$ & $\underline{118.46}$ \\
CSCS & 40.744 & 62.278 & 70.066 & 96.739 & 106.73 & 128.77 \\
CCCS & 42.928 & 69.997 & 71.914 & 102.84 & 120.35 & 130.73 \\
$\underline{\text { CCCC }}$ & $\underline{46.241}$ & $\underline{74.300}$ & $\underline{79.239}$ & $\underline{110.14}$ & $\underline{132.35}$ & $\underline{135.51}$ \\
\hline
\end{tabular}

Their identical results of six cases are underlined. As expected, the increase of the fundamental frequencies due to the curvature increase is basically same as in Table 4(a) and (b).

Finally, Table 6(a) and (b) list up the lowest six parameters of shallow hyperbolic paraboloidal shells with $a / R_{\mathrm{y}}=0.2$ and $a / R_{\mathrm{y}}=0.5$, respectively. The negative curvature ratio $\left(R_{\mathrm{x}} / R_{\mathrm{y}}=-1\right)$ indicates that the shell geometry is convex in one direction and concave in another direction. This geometric feature gives rise unusual response in frequency. For shells of spherical and cylindrical curvature, addition of curvature causes more stiffness in the structure, and it results in the increase of natural frequencies. But for shell of hyperbolic paraboloidal shell, addition of negative curvature causes the decrease of frequencies, when shell has free edges. For example, the SFFF shell gives $\Omega$ ${ }_{1}=6.648$ for $a / R_{\mathrm{x}}=0$ (plate), $\Omega_{1}=6.628$ for $a / R_{\mathrm{x}}=0.2$ and $\Omega_{1}=6.548$ for $a / R_{\mathrm{x}}=0.5$. As the constrained is increased along the edges, this tendency disappears.

\section{Conclusions}

The purpose of this paper was to present lists of accurate natural frequencies for free vibration of doubly curved, isotropic shallow shells of rectangular (square) planform under different sets of boundary conditions. For this purpose, mathematical procedure was described and accuracy of the present numerical solutions was well established by convergence and comparison studies. Thus, with strong background in solution accuracy, all the present frequency parameters were given in five
Table 6(a) Frequency parameters $\Omega$ of shallow hyperbolic paraboloidal shells, $R_{\mathrm{x}} / R_{\mathrm{y}}=-1, a / R_{\mathrm{y}}=0.2, a / b=1, a / h=20, v=0.3$.

\begin{tabular}{|c|c|c|c|c|c|c|}
\hline B.C. & $\Omega_{1}$ & $\Omega_{2}$ & $\Omega_{3}$ & $\Omega_{4}$ & $\Omega_{5}$ & $\Omega_{6}$ \\
\hline FFFF & 13.458 & 21.894 & 24.244 & 34.929 & 34.929 & 61.775 \\
\hline SFFF & 6.6281 & 16.057 & 25.414 & 26.762 & 48.931 & 50.889 \\
\hline CFFF & 3.8095 & 8.4752 & 22.356 & 27.892 & 30.617 & 44.083 \\
\hline SSFF & 3.3591 & 18.781 & 19.323 & 38.159 & 51.803 & 54.022 \\
\hline CSFF & 5.6190 & 20.369 & 25.086 & 43.345 & 53.237 & 54.290 \\
\hline CCFF & 7.0680 & 25.562 & 27.018 & 48.207 & 63.436 & 65.918 \\
\hline SFSF & 10.406 & 16.107 & 37.451 & 39.768 & 46.847 & 70.668 \\
\hline CFSF & 15.839 & 21.623 & 40.753 & 50.048 & 56.513 & 78.037 \\
\hline SSSF & 12.206 & 28.062 & 41.865 & 59.114 & 62.527 & 64.384 \\
\hline CSSF & 17.735 & 32.074 & 51.964 & 64.796 & 67.770 & 101.19 \\
\hline CCSF & 19.183 & 37.091 & 52.437 & 71.488 & 75.079 & 106.08 \\
\hline CFCF & 25.159 & 28.691 & 44.999 & 61.683 & 67.361 & 80.627 \\
\hline SCSF & 14.387 & 33.591 & 42.424 & 63.306 & 64.384 & 73.063 \\
\hline CSCF & 26.181 & 37.301 & 63.325 & 67.705 & 77.629 & 109.05 \\
\hline $\mathrm{CCCF}$ & 26.786 & 41.703 & 63.724 & 77.602 & 80.988 & 116.90 \\
\hline SSSS & 19.659 & 49.924 & 49.924 & 78.873 & 99.224 & 99.224 \\
\hline csss & 24.592 & 52.471 & 59.269 & 86.220 & 100.84 & 113.69 \\
\hline ccss & 28.542 & 61.369 & 61.547 & 93.043 & 115.07 & 115.19 \\
\hline cscs & 31.282 & 55.859 & 69.836 & 94.764 & 102.86 & 128.77 \\
\hline cccs & 34.232 & 64.379 & 71.753 & 101.07 & 116.93 & 130.85 \\
\hline $\operatorname{cccc}$ & 38.732 & 74.301 & 74.301 & 108.54 & 132.15 & 132.74 \\
\hline
\end{tabular}

Table $6(\mathrm{~b})$ Frequency parameters $\Omega$ of shallow hyperbolic paraboloidal shells, $R_{\mathrm{x}} / R_{\mathrm{y}}=-1, a / R_{\mathrm{y}}=0.5, a / b=1, a / h=20, v=0.3$.

\begin{tabular}{ccccccc}
\hline B.C. & $\Omega_{1}$ & $\Omega_{2}$ & $\Omega_{3}$ & $\Omega_{4}$ & $\Omega_{5}$ & $\Omega_{6}$ \\
\hline FFFF & 13.406 & 24.102 & 31.229 & 35.444 & 35.444 & 63.315 \\
SFFF & 6.5483 & 19.020 & 25.562 & 31.448 & 49.885 & 54.027 \\
CFFF & 4.8347 & 8.3111 & 25.101 & 29.276 & 32.576 & 46.547 \\
SSFF & 3.3096 & 19.414 & 24.886 & 37.875 & 55.594 & 56.620 \\
CSFF & 6.3397 & 24.220 & 28.365 & 44.741 & 54.552 & 56.821 \\
CCFF & 7.5551 & 28.612 & 32.675 & 51.171 & 67.028 & 67.642 \\
SFSF & 12.909 & 15.945 & 41.288 & 43.783 & 47.396 & 70.296 \\
CFSF & 18.435 & 26.084 & 45.659 & 53.025 & 57.756 & 79.27 \\
SSSF & 14.054 & 29.794 & 45.098 & 59.410 & 64.383 & 65.895 \\
CSSF & 21.710 & 36.488 & 54.729 & 68.581 & 69.133 & 101.62 \\
CCSF & 25.820 & 41.998 & 55.400 & 73.706 & 78.889 & 107.56 \\
CFCF & 36.944 & 38.401 & 51.656 & 64.224 & 68.305 & 84.713 \\
SCSF & 20.858 & 36.118 & 45.769 & 64.383 & 64.882 & 76.477 \\
CSCF & 37.483 & 45.199 & 65.551 & 72.410 & 78.955 & 110.01 \\
CCCF & 38.249 & 49.610 & 66.142 & 82.098 & 83.171 & 118.21 \\
SSSS & 19.252 & 52.805 & 52.805 & 78.438 & 101.94 & 101.94 \\
CSSS & 28.811 & 56.335 & 62.406 & 86.712 & 103.76 & 116.05 \\
CCSS & 35.016 & 65.275 & 65.497 & 94.178 & 117.69 & 117.73 \\
CSCS & 41.267 & 61.320 & 72.397 & 95.686 & 106.13 & 128.77 \\
CCCS & 44.601 & 69.579 & 75.114 & 102.50 & 119.86 & 133.46 \\
CCCC & 50.691 & 78.836 & 78.836 & 110.19 & 135.10 & 135.53 \\
& & & & & & \\
\hline
\end{tabular}


significant figures, while other previous literature provide four significant figures.

It is hoped that the present comprehensive sets of frequency parameters will serve as good reference for future comparison. Due to the space limitation, the present results were given only for the case of relatively thick case $(a / h=0.05)$ to complement Ref. [22]. In the next study, computation of frequency parameters will be done for very thin case $(a / h=0.01)$ to match with Ref. [21], and the effect of the shell thickness will be clarified.

\section{Acknowledgement}

The second author expresses his gratitude to the Japan Society for the Promotion of Science (JSPS) for the Funding Program MEXT/JSPS KAKENHI Grant Number 21K03957.

\section{References}

[1] Leissa AW, Vibration of Shells, NASA-SP-288, 1973.

[2] Soedel, W, Vibrations of Shells and Plates, CRC Press, (2004).

[3] The Japan Society of Mechanical Engineers, Handbook for Vibration and Buckling of Shells, Gihodo Shuppan Co., Ltd., Tokyo, 2003 (in Japanese).

[4] Narita D, Narita Y, Modelling of thin structural polymer composites for use of automobiles -review-, EPI Intl. J. Eng., 1 (2018), 13-20.

[5] Leissa AW, Kadi AS, Curvature effects on shallow shell vibrations, J. Sound Vibr., 16 (1971), 173-187.

[6] Leissa, AW, Lee, JK, Wang, AJ. Vibrations of cantilevered shallow cylindrical shells of rectangular planform, J. Sound Vib., 78 (1981), 311-228.

[7] Leissa AW, Lee JK, Wang AJ., Vibrations of cantilevered doubly curved shallow shells., Int. J. Solids Struct., 19 (1983), 411-424.

[8] Leissa AW, Narita Y, Vibrations of completely free shallow shells of rectangular planform, J. Sound Vibr., 96 (1984), 207-218.

[9] Narita Y, Leissa AW, Vibrations of corner point supported shallow shells of rectangular planform.

[22] Qatu MS, Asadi E, Vibration of doubly curved shallow shells with arbitrary boundaries, Applied Acous., 73 (2012), 21-27.
Earthq. Eng. Struct. Dynam., 12 (1984), 651-661.

[10] Qatu MS, Leissa AW. Effects of edge constraints upon shallow shell frequencies, Thin. Wall. Struct., 14 (1992), 347-379.

[11] Qatu MS, Review of shallow shell vibration research, Shock and Vibration Digest, 24 (1992), 3-15.

[12] Yu SD, Cleghorn WL, Fenton RG, On the accurate analysis of free vibration of open circular cylindrical shells, J. Sound Vibr., 188 (1995), 315-336.

[13] Liew KM, Lim CW, Vibratory behavior of doubly curved shallow shells of curvilinear planform, J. Eng. Mech., 121 (1995), 1277-1284.

[14] Liew KM, Lim CW, Vibration of doubly-curved shallow shells, Acta Mech., 114 (1996), 95-119.

[15] Lim CW, Liew KM, A pb-2 Ritz formulation for flexural vibration of shallow cylindrical shells of rectangular planform, J. Sound Vibr., 173 (1994), 343-375.

[16] Liew KM, Lim CW, Kitipornchai S, Vibration of shallow shells: a review with bibliography, Appl. Mech. Rev., 50 (1997), 431-444.

[17] Qatu MS, Recent research advances in the dynamic behavior of shells. Part 2: Homogeneous shells, Appl. Mech. Rev., 55 (2002), 415-434.

[18] Narita Y, Robinson P, Maximizing the fundamental frequency of laminated cylindrical panels using layerwise optimization, Intl. J. Mech. Sci., 48 (2006), 1516-1524.

[19] Monterrubio LE, Free vibration of shallow shells using the Rayleigh-Ritz method and penalty parameters, Proc. Inst. Mech. Engr., Part C: J. Mech. Eng. Sci., 23 (2009), 2263-2272.

[20] Qatu MS, Effect of inplane edge constraints on natural frequencies of simply supported doubly curved shallow shells. Thin Walled Struct., 49 (2011), $797-$ 803.

[21] Mochida Y, Ilanko S, Duke M, Narita Y, Free vibration analysis of doubly curved shallow shells using the Superposition-Galerkin method, J. Sound Vibr., 331 (2012), 1413-1425. 